\title{
Human blood microfluidic test chip for imaging, label-free biosensor
}

\author{
S. Jahns ${ }^{*}$, S. B. Gutekunst ${ }^{+}$, C. Selhuber-Unkel ${ }^{+}$, Y. Nazirizadeh ${ }^{*}$ and M. Gerken ${ }^{*}$ \\ *Institute of Electrical Engineering and Information Technology, \\ Christian-Albrechts-Universität zu Kiel, \\ Kaiserstrasse 2, \\ 24143 Kiel, Germany \\ Email address ${ }^{\dagger}$ : sja@tf.uni-kiel.de \\ Phone $^{\dagger}:+494318806255$ \\ Fax $^{\dagger}:+494318806253$ \\ ${ }^{+}$Institute for Materials Science, \\ Christian-Albrechts-Universität zu Kiel, \\ Kaiserstrasse 2, \\ 24143 Kiel, Germany
}

\begin{abstract}
We designed and evaluated a microfluidic test chip for human blood filtration and imaging label-free detection of multiple biomarkers. The microfluidic chip has a total size of $75 \mathrm{~mm} \times 25 \mathrm{~mm} \times 2 \mathrm{~mm}$. It is realized as an assembly of a plastic chip and a functionalized photonic crystal slab on a glass substrate. The plastic chip contains capillary channels, a filter membrane and a cavity open on one side. The photonic crystal chip is bonded with an adhesive foil to the open cavity. Human blood filtration is demonstrated. We determined that fluorescently labelled particles of diameter $3 \mu \mathrm{m}$ or larger are filtered out by the system. Refractometric measurements are performed with the test chip in combination with a compact imaging read-out system to investigate the system response to refractive index changes. Flow dynamics in the sensor cavity are imaged for replacing water by isopropanol. Finally, the binding of $500 \mathrm{nM}$ biotin dissolved in phosphate buffer saline to a photonic crystal surface locally functionalized with streptavidin is demonstrated.
\end{abstract}

\section{$1 \quad$ Introduction}

Compact, fast-operating, decentralized and easy-to-use sensors are of high interest for point-of-care applications. These requirements can be met by combining microfluidic technology with an integrated biosensor $[1,2]$. Microfluidic systems enable biochemical analyses using small reagent volumes [3]. Compared to conventional diagnostic devices in centralized facilities the small reagent volume needed in microfluidic systems is beneficial especially for human blood filtration and thus for biomarker analyses in blood [4]. Different concepts were introduced to separate plasma from whole blood such as special filter materials [5], designed filtration channels $[4,6]$, or separation via a membrane [7]. If a photonic crystal biosensor is integrated in such a microfluidic system, a label-free, compact and cost efficient system may be realized [2, 8, 9]. Photonic crystal slabs (PCS) are waveguides with a periodic nanostructure in a high refractive material supporting quasi-guided modes. These modes are visible as guided-mode resonances (GMRs) in the optical transmission and reflection spectrum. Because of the evanescent fraction of the quasi-guided modes propagating above the PCS surface, refractive index changes on the PCS surface result in a wavelength shift of the GMRs. When functionalizing the PCS surface with ligands, binding kinetics of the specific target proteins result in a refractive index change above the PCS surface and thus to a red-shift of the resonance wavelength. This can be tracked in real time with an optical read-out system [8-10]. Previously, optical read-out of the resonance spectral position was evaluated with a sensitive spectrometer for other microfluidic devices [9]. We developed an imaging read-out system, which converts the resulting shift of resonances in the transmission spectrum into an intensity change in the specific colour channel of a simple CMOS camera aligned to the resonance wavelength [11]. With such a system we are able to detect multiple biomarkers in parallel. In previous works we demonstrated the functionality of a labelfree photonic crystal biosensor and our imaging read-out system by successfully performing detections of $90 \mathrm{nM}$ CD40 ligand antibody as well as of $250 \mathrm{nM}$ streptavidin. Additionally, we suggested the concept of a microfluidic test chip for blood filtration and presented first binding experiments [12]. This system concept is depicted in figure 1 . Here, we investigate in detail the properties of the microfluidic test chip. First we evaluate the filter properties of our microfluidic test chip conducting experiments with whole blood as well as with fluorescently labelled particles of different diameters. Second, the flow characteristics in the cavity of the test chip are investigated by performing refractometric measurements with our imaging read-out system. Finally, spatially-resolved binding experiments are performed with the biotin-streptavidin system. 


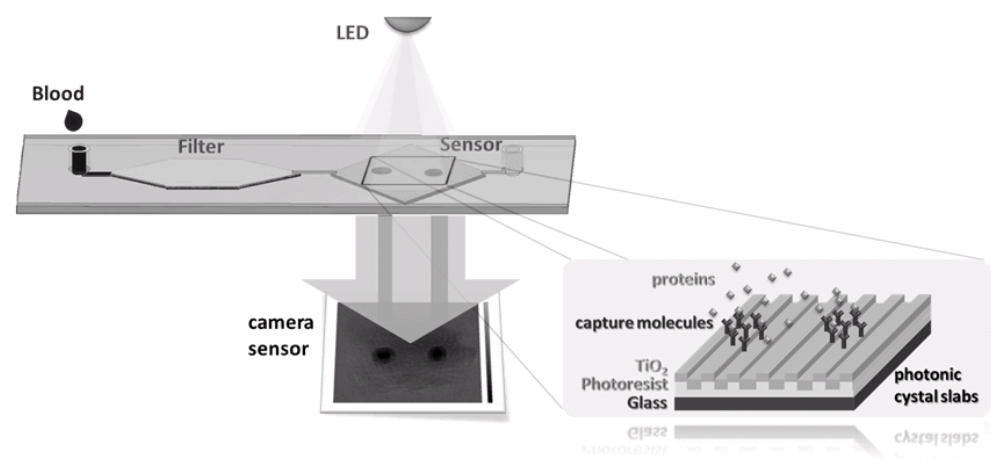

Figure 1: Schematic of microfluidic system for detection of multiple biomarkers with filter unit and bonded sensor chip. Labelfree read-out is performed with a compact imaging system and a camera.

\section{Results and Discussion}

\subsection{Test chip design and evaluation}

We designed a microfluidic test chip with a total size of $75 \mathrm{~mm}$ x $25 \mathrm{~mm}$ x $2 \mathrm{~mm}$. It is realized as an assembly of a plastic microfluidic chip and a functionalized photonic crystal slab on a glass substrate. The plastic microfluidic chip was developed in cooperation with the company Microfluidic ChipShop GmbH. As it is shown in figure $2 \mathrm{a}$, the plastic microfluidic chip consists of a main chip and a cover plate. The main chip includes a filler neck for the application of whole blood, capillary channels for blood guidance and a $15 \times 15 \mathrm{~mm}^{2}$ cavity. The cover plate includes a filter membrane for blood filtration and an adhesive foil for bonding on the photonic crystal sensor chip with an adhesive foil.
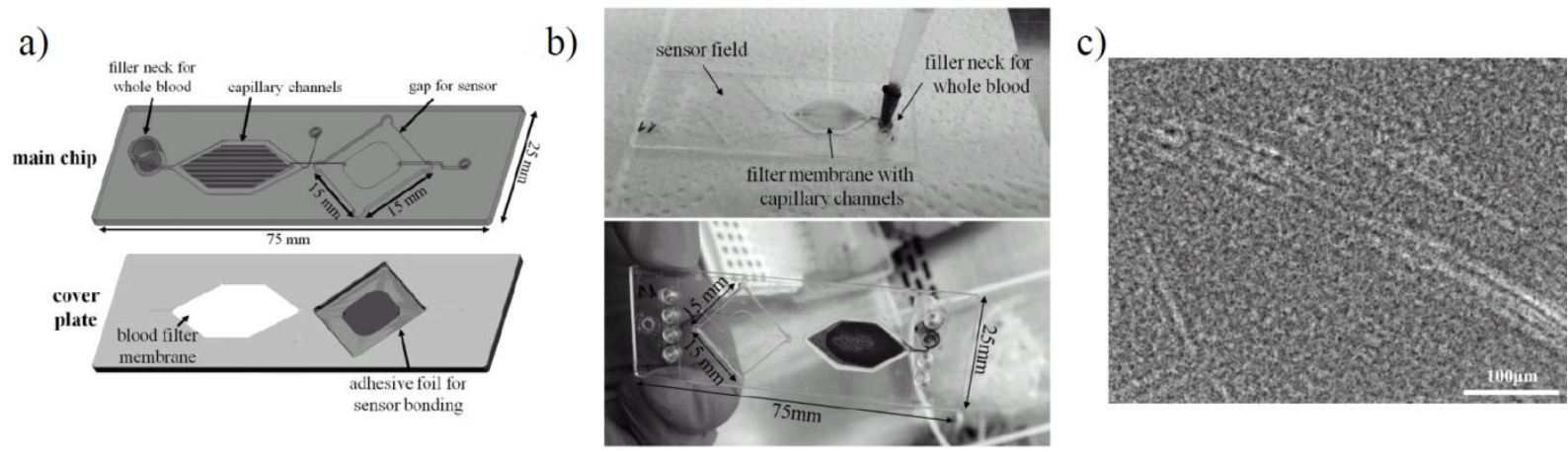

Figure 2: (a) Computer aided-design of a plastic microfluidic main chip including filler neck for whole blood, capillary channels and cavity for sensor (top) plus cover plate including blood filter membrane and adhesive foil for sensor bonding (bottom); (b) layout of the microfluidic test chip and human blood injection with a pipette through the filter; (c) microscope image of blood serum in the sensor cavity.

Blood filtration experiments were performed by injecting $200 \mu \mathrm{l}$ of whole blood spiked with the anticoagulant heparin to inhibit an immediate coagulation inside the capillary channels, which could appear due to surface enlargement. The red blood cells remain in the membrane and the clear blood serum (approx. 70-100 $\mu \mathrm{l}$ ) is transported to the sensor cavity, as seen in figures $2 b$. The microscopy image in figure $2 \mathrm{c}$ reveals that nevertheless a plenitude of blood components pass the filter and reach the cavity. This explains the schlieren observed previously in imaging detection with the photonic crystal sensor [12]. As larger particles deteriorate the label-free detection scheme, we next consider in detail, which particle sizes pass the filter.

For this purpose particles in different sizes (200 nm, $1 \mu \mathrm{m}, 2 \mu \mathrm{m}, 3 \mu \mathrm{m}$ and $5 \mu \mathrm{m}$ ) labelled with the fluorescence dyes DAPI (4',6-Diamidino-2-phenylindole), or FITC (Fluorescein isothiocyanate) were used. They were dissolved in doubled-distilled water. A glass substrate was bonded to the microfluidic plastic chip instead of the PCS sensor. Then, $200 \mu \mathrm{l}$ of the solution were injected with a pipette and pressed into the capillary channels and through the filter membrane. An image of the filtered solution reaching the sensor cavity was captured separately for each particle size with a fluorescence microscope. Exemplary, two recorded fluorescence images are shown for the particle sizes of $1 \mu \mathrm{m}$ (figure $3 \mathrm{a}$ ) and of $3 \mu \mathrm{m}$ (figure $3 \mathrm{~b}$ ). Figure $3 \mathrm{~b}$ shows that $3 \mu \mathrm{m}$ particles remain inside the filter membrane. However, the $200 \mathrm{~nm}$ and $1 \mu \mathrm{m}$ particles pass the filter and reach the sensor field. Additionally, some of the particles were labelled with acid or amino groups to check for chemical interactions with the filter membrane. No influence could be observed. In the next microfluidic chip generation it is desirable 
to filter out smaller particles to prevent schlieren that deteriorate the imaging measurement. Furthermore, a redesign of the sensor cavity is necessary.
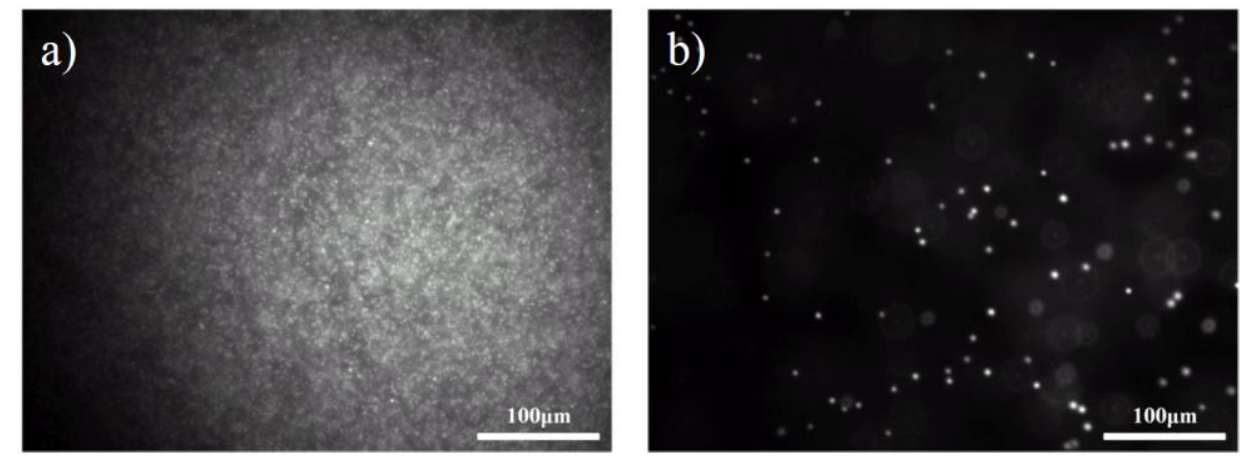

Figure 3: (a) Fluorescence photograph of $1 \mu \mathrm{m}$ large particles recorded in the sensor cavity; (b) fluorescence micrograph of $3 \mu \mathrm{m}$ large particles recorded in the filter membrane.

\subsection{Refractometric measurements and protein detection}

The aim is to perform a multiparameter test by local functionalization of the photonic crystal sensor chip and imaging readout. This test requires high homogeneity of the sensor signal across the sensor chip. Here, we investigate the signal homogeneity by performing imaging refractometric measurements. For this purpose a nonfunctionalized PCS was bonded into the microfluidic plastic chip and an analyte change from distilled water to isopropanol is conducted. As the quasi-guided mode samples the analyte refractive index, a change in the GMRs occurs. The test chip is placed in the imaging read-out system between two crossed linear polarizing filters and a parallelizing and focussing optic [12]. Because of this set-up only light interacting with the quasi-guided modes is transmitted through the crossed polarizers to the CMOS sensor of a simple camera [13]. An orange light emitting diode (LED) is used as excitation source and the resonance wavelength is aligned to the falling edge of the LED spectrum. Thus, a wavelength shift of the resonance is converted into an intensity reduction. The spatially-resolved transmission intensity is recorded by the CMOS camera sensor. During the measurement we acquired camera images of the sensor surface every 0.5 seconds. Here, only the green colour channel of the camera was read out with a LabVIEW program. Corresponding to the LED and the GMR spectrum the sensitivity of the green colour channel also has a falling edge in this spectral range. This enhances the intensity response to changes of the surrounding refractive index additionally. Figure 4a shows an example of a recorded image.

To generate a baseline signal the test chip was filled with water $\left(\mathrm{n}_{\mathrm{H} 2 \mathrm{O}}=1.33\right)$. After seven minutes the water was replaced by isopropanol $\left(\mathrm{n}_{\mathrm{iso}}=1.37\right)$. For figure $4 \mathrm{~b}$ the mean values of the two measuring fields (M1 \& M2) are calculated for each taken photograph and the resulting intensity values are plotted against time. For both signal sequences an intensity reduction of $\Delta \mathrm{I}=17.12 \%$ is observed after 15 minutes. We calculated a limit of detection (LOD) of around $7.3 \times 10^{-4}$ RIU. The LOD is defined as LOD $=((3 \times \sigma) / \Delta \mathrm{I}) \times \Delta \mathrm{n}$ with $\sigma$ as the standard deviation of the baseline signal, $\Delta \mathrm{I}$ as the intensity difference and $\Delta \mathrm{n}$ as the refractive index difference of the analytes.

a)

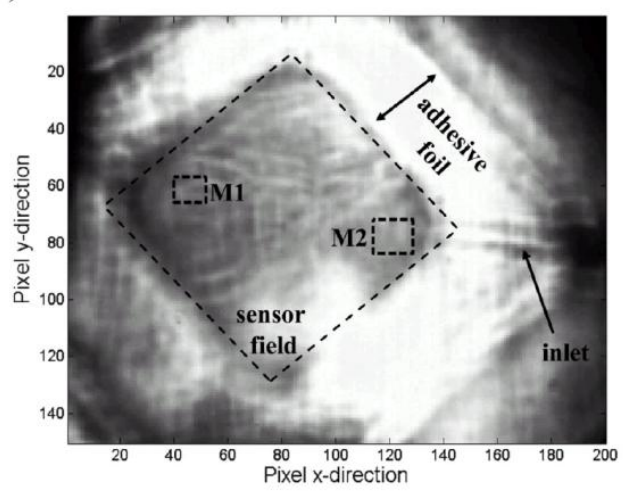

b)

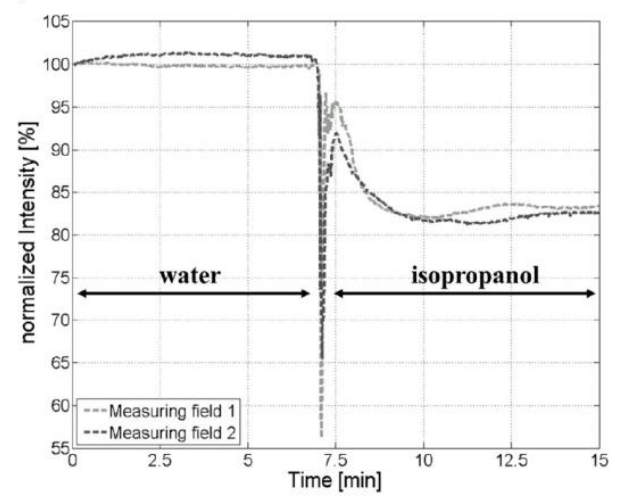

Figure 4: (a) Recorded photograph of the sensor field with the imaging read-out system. The sensor field and measuring fields M1 and M2 are marked with black dashed rectangles. The adhesive foil as well as the inlet channel can be seen on this picture; (b) the mean value within the measuring fields is calculated for each photograph and the intensity values are plotted against time. 
To investigate the homogeneity of the signal, the first recorded photograph is subtracted from every following photograph and the difference intensity images are analysed as a movie. Figure 5 depicts six examples of difference images with the above used measuring fields marked again as black dashed rectangles. In the movie it is observed that already before the isopropanol injection intensity differences are recorded around the measuring field M2. These are seen in the difference image at 2.02 minutes shown in figure 5. We attribute these changes to mechanical stress deformations of the microfluidic test chip. The isopropanol is guided via the inlet into the sensor field and reaches the field at 6.8 minutes. Figure 5 shows that already at 7.04 minutes (14 seconds after isopropanol injection) a refractive index change is observed across the entire sensor cavity. For the next 40 seconds rapid fluctuations of the difference intensity image are observed that may be attributed to flow dynamics. After approximately 1 minute a steady state is achieved in the top-right half of the test chip. The images at 7.70 minutes and 15.0 minutes are similar in this region. On the bottom left on the other hand, further intensity changes are observed. Visually, these could be attributed to the formation of an air bubble. This leads to the assumption that the bonding adhesive is not stable against isopropanol. Instead of isopropanol glycerol may be used for system verification in the future. Furthermore, additional bubble formation is observed at the inlet. Figure 6 presents difference images at different times showing the rapid change in intensity upon injection and the much smaller changes before and after injection. The intensity reduction at the upper edge of the sensor field observed in figure $6 \mathrm{c}$ occurs from air accumulation caused by the rise of air bubbles.

Considering the difference image in figure 5 at 15 minutes, highly inhomogeneous intensity changes are observed within the sensor field. As isopropanol has a homogeneous refractive index, we expected to observe an intensity reduction distributed homogeneously over the whole sensor field and thus a shift of the distribution towards lower grey scale values. We attribute this inhomogeneity to mechanical deformation upon injection of isopropanol. This behaviour is highly detrimental for an imaging biosensor and should be prevented by a more stable microfluidic test chip layout in the next design generation. Therefore, a more stable mechanical design of the chip is targeted in the next generation. This may be achieved by thicker substrates. Additionally, the large square cavity may be replaced by a meandering channel that is better supported by sidewalls. For such an approach the dwell time of the biomarkers on top of the sensor fields need to be optimized.
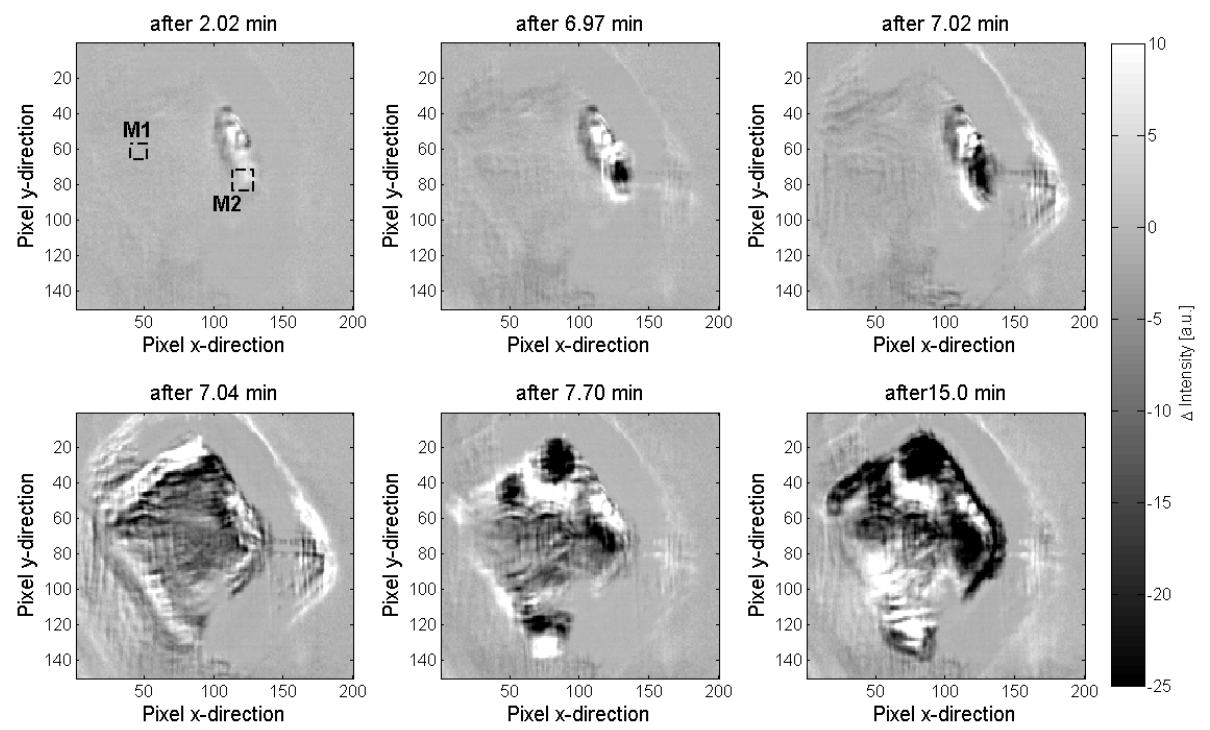

Figure 5: Examples of intensity difference images with the first photograph subtracted for each investigated time. Isopropanol injection occurs at 7 min close to measuring field $\mathrm{M} 2$. 
a)

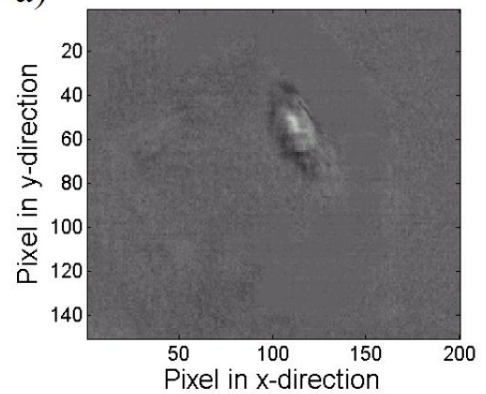

b)

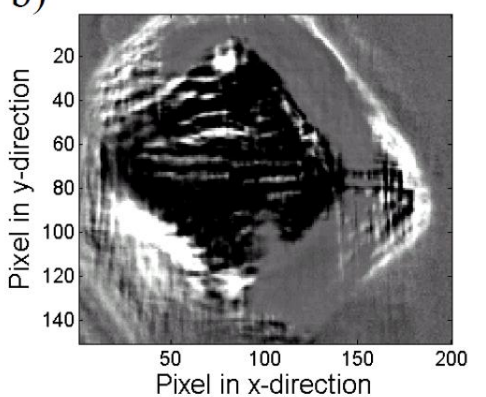

c)

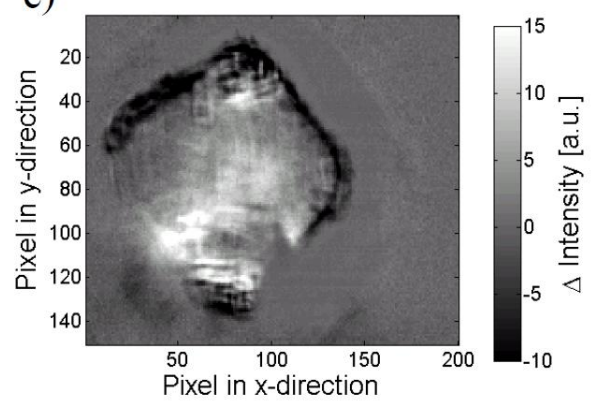

Figure 6: Intensity difference images between different points in time. (a) Image at 4.80 min minus the one at 2.23 min. (b) Image at 7.20 min minus the one at $6.74 \mathrm{~min}$. (c) Image at $13.32 \mathrm{~min}$ minus the one at $10.55 \mathrm{~min}$

Nevertheless, we performed first binding experiments by immobilizing initially two drops of $1.00 \mu \mathrm{M}$ of biotinylated bovine serum albumin (BSA-biotin) and then $1.00 \mu \mathrm{M}$ of streptavidin via covalent coupling on the surface of the photonic crystal [12]. Afterwards, $500 \mathrm{nM}$ BSA-biotin dissolved in phosphate buffer saline were injected into the test chip to simulate measurement conditions as they exist for binding experiments with whole blood. The test chip was placed inside the imaging read-out system and images were taken continuously during the protein detection (figure 7a). For the two functionalized fields as well as for a non-functionalized reference field the integrated intensity is plotted normalized to the start value (figure 7b). For the measuring fields a signal decay is observed as is expected. The imaging detection in figure 7 a reveals the inhomogeneous intensity behaviour across the sensor chip requiring careful consideration of these results.

a)

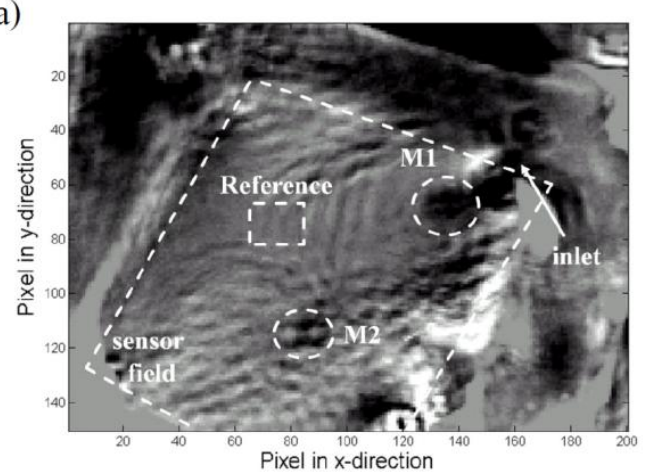

b)

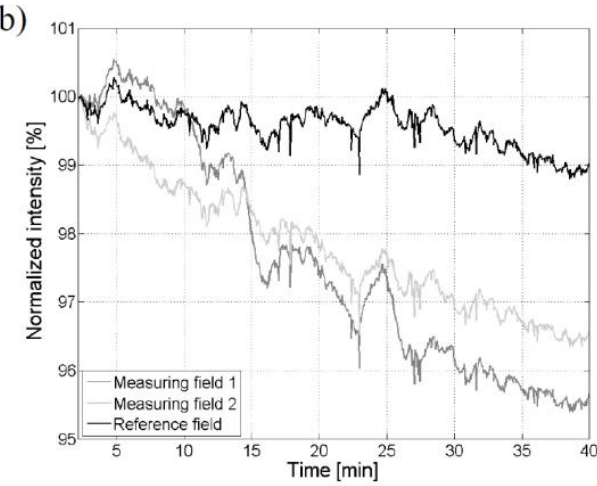

Figure 7: (a) Recorded photograph of the sensor field with the imaging read-out system at 40 minutes after injection of BSAbiotin. The sensor field and measuring fields M1 and M2 and the reference are marked white dashed; (b) the mean value of the normalized intensities within the measuring and reference fields is calculated for each taken photograph and the intensity values are plotted against time

\section{Conclusion}

We designed and evaluated a microfluidic test chip for measuring biomarkers from whole blood. Whole blood filtration was performed successfully with only clear blood serum reaching the sensor cavity. Fluorescence measurements revealed that polymer particles with sizes larger than $3 \mu \mathrm{m}$ remain in the filter. Imaging with our photonic crystal sensor yielded that even though a steady state is reached after approximately 40 seconds after an analyte exchange, the observed signal intensities are highly inhomogeneous. We attribute this to mechanical deformation of the microfluidic test chip upon fluid injection. Nevertheless, we obtained an LOD of $7.3 \times 10^{-4} \mathrm{RIU}$ by performing refractometric measurements. Additionally, with the current sensor design the local binding of $500 \mathrm{nM}$ BSA-biotin dissolved in phosphate buffer saline could be observed for two binding positions although with high signal noise. In conclusion, the approach of using a disposable microfluidic test chip and an imaging read-out system is promising for multiple protein detection in human blood in point-of-care applications. The presented results indicate how the design should be improved in the next-generation chip for sensitive detection.

\section{$4 \quad$ Acknowledgement}

The authors acknowledge support by the European Research Council within the project Photosmart (307800) and by the German Federal Ministry of Education and Research (BMBF) within the project BioCard 
(0316145A). S. B. Gutekunst and C. Selhuber-Unkel acknowledge support from the DFG through the Emmy Noether program (grant SE-1801/2-1).

\section{$5 \quad$ References}

[1] C. Liu, D. Cui, H. Li, "A hard-soft microfluidic biosensor flow cell for SPR imaging application", Biosensors and Bioelectronics, vol. 26, pp. 225-261 (2010).

[2] C. J. Choi, I. D. Block, B. Bole, D. Dralle, B. T. Cunningham, "Label-Free Photonic Crystal Biosensor Integrated Microfluidic Chip for Detemination of Kinetic Reaction Rate Constants," IEE Sensors Journal, vol. 9, pp. 1697-1704 (2009).

[3] D. Janasek, J. Franzke, A. Manz, "Scaling and the design of miniaturized chemical-analysis systems," Nature, vol. 442, pp. 374-380 (2006)

[4] T. A. Crowley, V. Pizziconi, "Isolation of plasma from whole blood using planar microfilters for labon-a-chip applications," Lab Chip, vol. 5, pp. 922-929 (2005).

[5] L. M. Bananno, L. A. DeLouise, "Whole blood optical biosensor", Biosensors and Bioelectronics," vol. 23, pp. 444-448 (2007).

[6] J.-N Kuo, Y.-H. Zhan, "Microfluidic chip for rapid and automatic extraction of plasma from whole blood," Microsystem Technology, vol. 21, pp. 255-261 (2015).

[7] C. Liu, M. Mauk, R. Gross, F. D. Bushman, P. H. Edelstein, "Membrane-based, Sedimentation-Assited Plasma Separator for Point-of-Care Applications," Analytical Chemistry, vol. 85, pp. 10463-10470 (2013).

[8] R. Magnusson, D. Wawro, S. Zimmermann, Y. Ding, "Resonant Photonic Biosensor with PolarizationBased Multiparametric Discimination in Each Channel" Sensors, vol. 11, pp. 1476-1488 (2001).

[9] D. Threm, Y. Nazirizadeh, M. Gerken, "Photonic Crystal Biosensors towards On-Chip Integration," Journal of Biophotonics, vol. 5, issue 8 - 9, pp. 601 - 616 (2012).

[10] L. Sheng- Fu, D. Ting- Jou, L. Jen- Tsai, L. Chien- Chieh, Y. Tsung-Hsun, C. Wen- Yih, C. JenqYang, "A Guided Mode Resonance Aptasensor for Thrombin Detection," Sensors, vol. 11, no. 9, pp. 8953-8965, 2011.

[11] S. Jahns, Y. Nazirizadeh, B. Meyer, S. B. Gutekunst, C. Selhuber-Unkel, M. Gerken, "Photometric Aptasensor using Biofunctionalized Photonic Crystal Slabs," Proc. IEEE Sensors 2013, Baltimore (USA) (2013).

[12] S. Jahns, P. Glorius, M. Hansen, Y. Nazirizadeh and M. Gerken, "Imaging label-free biosensor with microfluidic system", Proc. SPIE, vol. 9518, p. 95180K-95180K-6 (2015)

[13] Y. Nazirizadeh, U. Bog, S. Sekula, T. Mappes, U. Lemmer, M. Gerken, "Low-cost label-free biosensors using photonic crystals embedded between crossed polarizers", Optics Express 18, 19120-19128 (2010) 